\title{
Hair growth promoting effect of dermal papilla like tissues from canine adipose- derived mesenchymal stem cells through vascular endothelial growth factor
}

\author{
Aeri LEE ${ }^{1)}$, Sohee $\mathrm{BAE}^{1)}$, Seung Hoon LEE ${ }^{1)}$, Oh-Kyeong KWEON ${ }^{1)}$ and Wan Hee $\mathrm{KIM}^{1) *}$ \\ 1) Department of Veterinary Clinical Sciences, College of Veterinary Medicine and Research Institute for Veterinary Science, Seoul \\ National University, 1, Gwanak-ro, Gwanak-gu, Seoul 09926, Republic of Korea
}

(Received 9 March 2016/Accepted 26 August 2016/Published online in J-STAGE 16 September 2016)

ABSTRACT. The purpose of this study was to investigate the protein expression pattern and the in vivo trichogenicity of dermal papilla-like tissues (DPLTs) made from canine adipose-derived mesenchymal stem cells (ASCs) in athymic nude mice. Canine ASCs were isolated and cultured from adipose tissue, and differentiation was induced by culturing ASCs in dermal papilla forming media. DPLTs were embedded in collagen gel, and their structural characteristics and protein expression were evaluated by hematoxylin and eosin stain and immunohistochemistry. Athymic nude mice were divided into two groups (control and DPLTs groups), and DPLTs were injected in skin wounds of mice in the DPLTs group. The trichogenicity of DPLTs was assessed by gross and histological evaluations for 30 days. The fate and the growth factor-secretion effect of DPLTs were evaluated by immunohistochemistry and Western blotting. DPLTs have a compact aggregated structure, form extracellular matrix and highly express the protein specific for dermal papillae, including ALP and versican. New hair follicle formation was remarkable in nude mice of the DPLTs group in gross findings and H\&E stain. Vascularization was increased in the DPLTs group, which was the effect of vascular endothelial growth factor secreted by DPLTs in vitro and in vivo. These data suggest that engineered canine DPLTs have characteristics of dermal papillae and have a positive effect on hair regeneration by secreting growth factors. KEY WORDS: adipose-derived mesenchymal stem cell, athymic nude mouse, dermal papilla-like tissue, hair regeneration, vascular endothelial growth factor

doi: 10.1292/jvms.16-0122; J. Vet. Med. Sci. 78(12): 1811-1818, 2016

Alopecia is the loss of hairs from some of all areas of the body. Hair loss is one of the major concerns in veterinary as well as medical dermatology fields, and several therapeutic options have been evaluated and applied for both human and animal patients. Current treatments, such as hair implantation surgery and medication, have many limitations, and in vitro culturing or bioengineering of cells from hair follicles has taken center stage as an alternative treatment strategy $[3,22,32]$. Among various sources for regenerative medicine, adipose derived mesenchymal stem cells (ASCs) are one of the most valuable stem cell populations, because adipose tissues can be obtained noninvasively and in large quantities [22].

The development of hair follicles relies on interactions between the epidermis and dermis, i.e., epidermal-mesenchymal interaction (EMI) [27]. Dermal papilla cells and dermal sheath cells are the main components of the mesenchymal cell population $[28,30]$. Among these, dermal papillae (DPs) play an important role in the EMI cascade, hair follicle formation, maintenance of hair growth and hair cycle control [5, 8, 28, 30]. More recently, it is elucidated that DP

\footnotetext{
*Correspondence to: Kim, W.H., Department of Veterinary Clinical Sciences, College of Veterinary Medicine and Research Institute for Veterinary Science, Seoul National University, 1, Gwanak-ro, Gwanak-gu, Seoul 09926, Republic of Korea.

e-mail: whkim@snu.ac.kr

(C)2016 The Japanese Society of Veterinary Science

This is an open-access article distributed under the terms of the Creative Commons Attribution Non-Commercial No Derivatives (by-nc-nd) License $<$ http://creativecommons.org/licenses/by-nc-nd/4.0/>.
}

also can be a reservoir of multipotent stem cells and retain the information to determine hair fiber type [7,9]. Therefore, in vitro culture or reconstruction of DPs is an important issue in hair regeneration therapy. However, DP isolation from hair follicle is technically difficult and time-consuming, and cultured dermal papilla cells (DPCs) lose their trichogenicity after few passages [25]. To overcome these limitations, reconstructed dermal papilla like tissues (DPLTs) from easily obtained ASCs could be a useful alternative for cultured DPs [32].

Our laboratory group has recently reconstructed DPLTs from canine ASCs and reported that they had similar expression patterns as canine DPCs at the mRNA and protein levels [1]. In the present study, we reconstructed DPLTs from canine ASCs and characterized them by H\&E stain and immunohistochemistry. In vivo trichogenicity of DPLTs was investigated by injecting DPLTs in skin wounds of athymic nude mice. We observed the status of the wound bed by gross and histological evaluations, and the role of DPLTs in this process was evaluated by immunohistochemistry and Western blotting. We hypothesized that DPLTs have an ability to regenerate hair follicles as a substitute of DPs when implanted on skin of athymic nude mice, as well as have similar structure and protein expression pattern with DPs.

\section{MATERIALS AND METHODS}

Isolation and cultivation of adipose derived mesenchymal stem cells: The canine ASCs used in this study were obtained in the same manner described in previous paper, and these were previously characterized [26]. Briefly, adipose tissues 
were aseptically harvested from gluteal region of 2-year-old beagle dogs, which were sacrificed for other objectives. The procedure was conducted with the approval of the Institutional Animal Care and Use Committee of Seoul National University (SNU-120306-5). Adipose tissues were washed extensively with phosphate-buffered saline (PBS), minced with cut and digested with collagenase type $\mathrm{I}(1 \mathrm{mg} / \mathrm{m}$; Sigma-Aldrich, St. Louis, MO, U.S.A.) for $2 \mathrm{hr}$ at $37^{\circ} \mathrm{C}$. The tissue samples were washed with PBS solution and centrifuged at $300 \times \mathrm{g}$ for $10 \mathrm{~min}$. The pellets were resuspended, filtered with a $100 \mu \mathrm{m}$ nylon mesh and incubated overnight in low-glucose Dulbecco's modified eagle medium (low glucose-DMEM, Gibco ${ }^{\circledR}$, Life Technologies, Grand Island, NY, U.S.A.) supplemented with inactivated $10 \%$ fetal bovine serum (FBS, Gibco ${ }^{\circledR}$, Life Technologies) and 100 units/ $\mathrm{m} l$ penicillin- $100 \mathrm{mg} / \mathrm{m} l$ streptomycin at $37.0^{\circ} \mathrm{C}$, in a $5 \%$ $\mathrm{CO}_{2}$ incubator. After $24 \mathrm{hr}$, unattached cells and residual non-adherent red blood cells were removed by washing with PBS solution. The medium was changed every 2 days until the cells reached $80-90 \%$ confluence. At passage 3, the cells were used for the following experiments.

Reconstruction of dermal papilla-like tissues: After canine ASCs were cultured until $80 \%$ confluence, the culture medium was changed from low glucose-DMEM to dermal papilla forming media (DPFM) [32] that contained $10 \mathrm{ng} / \mathrm{m} l$ hydrocortisone (Hydrocortisone, Sigma-Aldrich), $5 \mathrm{ml} \mathrm{In}-$ sulin-Transferrin-Selenium liquid media (ITS, Gibco ${ }^{\circledR}$, Life Technologies), 100 units $/ \mathrm{m} l$ penicillin, $100 \mathrm{mg} / \mathrm{m} l$ streptomycin and $20 \mathrm{ng} / \mathrm{ml}$ recombinant human hepatocyte growth factor (HGF, Gibco ${ }^{\circledR}$, Life Technologies). The medium was changed once every 3 days for three weeks. The last changed medium contained green fluorescent nanoparticles (CellStalker II-CSF, Biterials, Seoul, Korea) at concentration of $0.2 \mathrm{mg} / \mathrm{m} l$ to label the cells. After that, the cultured cells were treated with the cell dissociation reagent (Accutase, Gibco, Life Technologies) to detach the cells from the culture dish (detach-attach step). Cells were re-applied at concentrations of $8 \times 10^{4}$ cells $/ \mathrm{cm}^{2}$. Cells were undergone redistribution, and aggregation appeared $24 \mathrm{hr}$ after the detach-attach step. Cell aggregates became suspended $24-48 \mathrm{hr}$ and then isolated from the culture medium by centrifugation at $500 \mathrm{rpm}$ for $3 \mathrm{~min}$. All procedures used for reconstruction of DPLTs were based on and modified from previous reports [32].

Immunohistochemistry of DPLTs: To observe the structure and protein expression state of DPLTs, they were harvested and embedded in collagen gel. According to the manufacturer's instructions, seven parts of $0.5 \%$ collagen solution (Matrixen-PSC, Bioland Ltd., Gyeonggi, Korea), two parts of 5X DMEM (Gibco, Life Technologies) and one part of $0.05 \mathrm{~N} \mathrm{NaOH}$ solution containing $2.2 \%$ sodium bicarbonate and $200 \mathrm{mM}$ HEPES were mixed and blended with reconstructed DPLTs on ice. This mixture was then placed in a humidified incubator $\left(95 \%\right.$ air $/ 5 \% \mathrm{CO}_{2}$ at $\left.37^{\circ} \mathrm{C}\right)$ for $1 \mathrm{hr}$ until gelation. After the gel containing DPLTs was fixed with $4 \%$ paraformaldehyde solution overnight, that was embedded in paraffin. Sections were cut in $4 \mu \mathrm{m}$ and stained with hematoxylin and eosin (H\&E). Immunohistochemistry for laminin (rabbit polyclonal antibody, ab11575, 1:100, Abcam,
Cambridge, MA, U.S.A.), vimentin (mouse monoclonal antibody, ab8069, 1:100, Abcam), versican (rabbit polyclonal antibody, ab19345, 1:50, Abcam) and vascular endothelial growth factor (rabbit polyclonal antibody, ab46154, 1:100, Abcam) were performed. The sections were exposed to horseradish peroxidase (HRP)-conjugated goat anti-rabbit IgG (G21234, 1:500, Molecular Probes ${ }^{\mathrm{TM}}$, Eugene, OR, U.S.A.) or goat anti-mouse IgG (G21040, 1:500, Molecular Probes ${ }^{\mathrm{TM}}$ ) and were visualized by a reaction with $3,3^{\prime}$-diaminobenzidine (DAB) tetrahydrochloride (ImmPACT DAB peroxidase substrate, SK-4105, Vector Laboratories, Burlingame, CA, U.S.A.). Canine skin tissue was used as the positive control, and 5\% normal goat serum was applied instead of primary antibodies as the negative control.

Alkaline phosphatase activity: Alkaline phosphatase (ALP) activity of ASC and DPLTs was measured with a colorimetric method using a TRACP \& ALP assay kit (\#MK301, Takara Bio, Tokyo, Japan). Undifferentiated ASCs were washed with $0.9 \%$ sodium chloride, and floating DPLTs were collected with culture medium and washed. Proteins were extracted by sonication in the extraction solution included in the kit. Equal parts of the cell lysate and substrate solution containing $p$-nitro-phenyl phosphate (pNPP) were mixed and reacted in a 96-well plate for 30 min. Absorbance at $415 \mathrm{~nm}$ of each well was measured with a microplate reader (Model 680, Bio-Rad Laboratories, Burlingame, CA, U.S.A.). Protein concentration in the cell lysate was measured at the same time with a Bradford assay for normalizing the ALP activity.

Preparation of the experimental animal model: The hair inducing activity of DPLTs and their fate were evaluated in vivo. Thirty athymic nude mice (BALB/cSlc-nu/nu, six weeks old, female, 17-20 g, Japan SLC Inc., Shizuoka, Japan) were used in this study, which were divided equally in each group (control or DPLTs group; 10, 20 and 30 days). Mice were anesthetized by intraperitoneal injection of a tiletamine/zolazepam combination (30 mg/kg, Zoletil50, Virbac Korea, Seoul, Korea) and xylazine $(10 \mathrm{mg} / \mathrm{kg}$, Rompun, Bayer Korea, Seoul, Korea). The scalp of mice was prepared aseptically, and full thickness skin wounds were created with a $6 \mathrm{~mm}$ biopsy punch. In the DPLTs group, 400 DPLTs mixed with $50 \mu l$ of PBS were injected into the inner-dermis at the border between the wound and the normal skin. On the other hand, only $50 \mu l$ of PBS were injected in the same manner to mice in the control group. Tegaderm (3M Health Care, St. Paul, MN, U.S.A.) was used for wound protection until wound healing was completed. The animals were housed in a specific pathogen-free (SPF) room at Seoul National University, and all of the procedures were approved by the Institutional Animal Care and Use Committee of Seoul National University (SNU-140917-2-3).

Evaluation of hair growth in gross and histological examinations: The wounds were photographed using a digital camera at $0,10,20$ and 30 days after transplantation of DPLTs. The mice were euthanized by cervical dislocation at 10, 20 and 30 days from the time of DPLTs injection. The skin around the wounds was harvested and divided into two parts with wound as a center: one part for histo- 
logic evaluation and the other part for Western blotting. The sampled skin was fixed in 10\% neutral formalin solution and embedded in paraffin. Routine histological processing and H\&E stain were performed. Histological examination was performed in a blinded fashion under light microscopy. The number of hair follicles and sebaceous glands in the regenerated wounds, and vascularization were evaluated. Immunohistochemistry for endothelial cells with anti-CD31 antibody (rabbit polyclonal antibody, ab28364, 1:50, Abcam) was used to visualize the vessels. The positive control (murine skin tissues) and negative control were also applied in the same manner. Visualization and detection procedures were conducted in the same manner as previously described. Vascularization was evaluated by the number of blood vessels in the high-power field $(\times 400)$ using five randomly selected fields from each skin section. For immunofluorescence, the sections were incubated with rabbit anti-vascular endothelial growth factor (VEGF) antibody (ab46154, 1:200, Abcam). Then, CyTM3-conjugated AffiniPure donkey anti-rabbit IgG $(\mathrm{H}+\mathrm{L})$ (711-165-152, 1:500, Jackson ImmunoResearch, West Grove, PA, U.S.A.) was applied at room temperature for $1 \mathrm{hr}$. DAPI (4,6-diamidino-2-phenylindole) was used for nuclear counter staining.

Western blotting: The tissue extracts were prepared from the skin with the PRO-PREPTM protein extraction solution (iNtRON Biotechnology, Gyonggi-do, Korea). The protein concentrations were determined with the Bradford assay (Bio-Rad Laboratories). The extracted proteins (20 $\mu \mathrm{g})$ were mixed with Laemmli's sodium dodecyl sulfate (SDS)-sample buffer (GenDEPOT, Baker, TX, U.S.A.) and boiled for $5 \mathrm{~min}$ before loading. Samples were run on a $12 \%$ SDS-polyacrylamide gel and transferred onto a nitrocellulose membrane. The membranes were blocked with 5\% skim milk for $1 \mathrm{hr}$ at room temperature and incubated overnight at $4^{\circ} \mathrm{C}$ with rabbit polyclonal anti-VEGF antibody (ab46154, 1:1,000, Abcam). Anti-rabbit IgG-HRP was used as a secondary antibody for $1 \mathrm{hr}$ at room temperature. Anti- $\beta$-actin antibody (sc-47778, 1:1,000, Santa Cruz Biotechnology, Santa Gruz, CA, U.S.A.) was used for a loading control. Blots were visualized with an enhanced chemiluminescence kit (ECL Kit, Invitrogen ${ }^{\mathrm{TM}}$, Life Technologies) and visualized under ChemiDoc. The optical densities were quantified using image analyzer software (Image J, U.A. National Institutes of Health, Bethesda, MD, U.S.A.).

Statistical analysis: Statistical analyses were performed using SPSS, version 22.0 (SPSS Inc., Tokyo, Japan). A Mann-Whitney U test was used to assess the changes or differences between the control group and the DPLTs group. A $p$-value $<0.05$ was considered statistically significant. Data are reported as the mean values, with the variability expressed as standard deviation.

\section{RESULTS}

Reconstruction of DPLTs: ASCs underwent morphological changes by DPFM from a spindle shape to a polygonal shape, which started approximately $24 \mathrm{hr}$ after changing media. After the detach-attachment step, cell distribution was changed, and groups of cells began to pull apart and form aggregates; cell aggregates started to float after 24-48 hr. The morphology and the size of DPLTs were consistent with the previous study $[1,32]$.

Structure and protein expression of DPLTs: The compact structure of the DPLTs, which had closely connected cells, was observed by H\&E staining (Fig. 1A). There were no regions of necrosis or deformed cells in the aggregated structure. Collagen-embedded DPLTs were immunostained by versican, laminin and vimentin (Fig. 1B-1D). In addition, intracellular ALP concentration was measured in the DPLTs and ASCs. Reconstructed self-aggregated DPLTs had significantly higher levels of ALP compared to the ASCs (Fig. 1E).

In vivo hair inductivity: Approximately 10 days after wound induction, wound healing was completed in all groups. New hair fibers on the regenerated skin were observed at 15-20 days after injection, especially in the DPLTs group (Fig. 2). Regenerated hairs at the wound bed in the control group were short and thin as well as mainly observed around the periphery of the wound, whereas the central wound region had regenerated hairs in the DPLTs group. Intriguingly, the number of hairs at the wound bed slightly decreased at day 30 .

The difference between the groups was more apparent in the histological evaluation. At day 10 and day 20, the skin tissues from the DPLTs group (4.6 \pm 2.19 and $10.7 \pm 2.89$ / wound bed) contained significantly more regenerated hair follicles in the wound bed than those from the control group $(0.5 \pm 1.00$ and $4.0 \pm 1.00$ /wound bed) (Fig. 3A and 3B) Newly formed hair follicles increased until day 20, and then, the trends changed by 30 days. The number of regenerated hair follicles was slightly decreased in both groups, and the difference between two groups was not significant at day 30 .

The number of sebaceous glands in the wound bed observed at days 10 and 20 was significantly higher in the DPLTs group ( $2.2 \pm 0.84$ and $2.3 \pm 0.50$ /wound bed) compared to the control group $(0.0 \pm 0.00$ and $0.3 \pm 0.50$ /wound bed) (Fig. 3A and 3C). However, similar to the hair follicles, there was no significant difference between two groups in the number of sebaceous glands at day 30 .

In addition, the majority of the pre-existing hair follicles outside the wound bed were in the anagen phase at day 20 , which consisted of multilayered cells and located deeply in the subcutaneous region. While, most pre-existing hair follicles at day 30 were in the telogen phase (Fig. 3A).

Angiogenesis was evaluated by counting the number of vessels in the slides, which were immunostained against CD31 at day 10 (Fig. 4). The number of CD31-positive vessels at the wound per five fields was significantly higher in the DPLTs group $(48.0 \pm 15.05 / \mathrm{HPF})$ relative to the control group $(16.2 \pm 5.54 / \mathrm{HPF})$ at day 10 . The difference between groups decreased at days 20 and 30 .

The role of DPLTs in hair inductivity: Under fluorescence microscopy, it is confirmed that injected fluorescencelabeled DPLTs did not constitute regenerated DPs. Instead of structural function, injected DPLTs were located in subcutaneous region. 
A

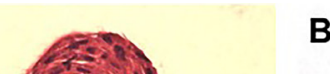

B

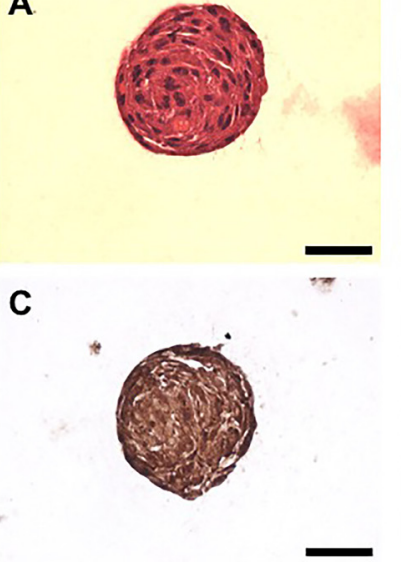

E

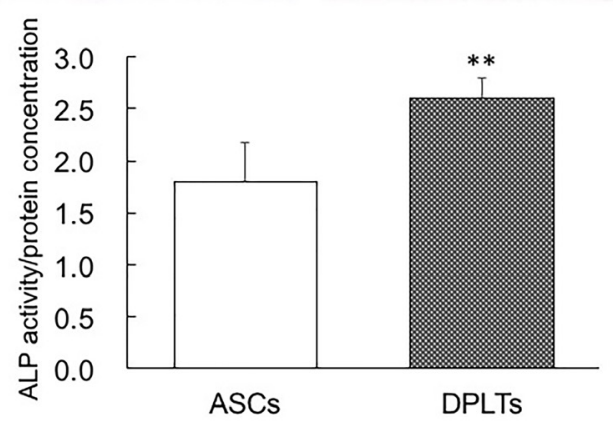

Fig. 1. Analysis of the structure and the protein expression of collagen embedded DPLTs by histological (A), immunohistochemical (IHC) staining (B-D) and alkaline phosphatase activity (E). (A) Notice compact aggregated structure of the DPLTs. H\&E stain. DPLTs were immunostained by versican (B), laminin (C) and vimentin (D). bar=50 $\mu \mathrm{m}$ in all panels. (E) Intracellular ALP concentration was significantly $(* * P<0.01)$ higher in DPLTs compared to ASCs. ALP activity normalized by protein concentration of ASCs and DPLTs.

We performed immunohistochemistry on collagenembedded DPLTs and mice skin tissues at day 10 in order to identify the paracrine effect of DPLTs on hair regeneration. Several VEGF-positive DPLTs were detected in both samples, which indicates that DPLTs could secrete VEGF in vitro and in vivo (Fig. 5A and 5B). Western blotting was performed to quantify the expression level of VEGF at day 10. The larger amount of VEGF was detected in the DPLTs group compared with the control group, though the difference in the thickness of the bands was not significant (Fig. 5C).

\section{DISCUSSION}

Our previous study [1] demonstrated that DPLTs could be successfully reconstructed from canine ASCs. In succession, reconstructed DPLTs were evaluated as an alternative to actual DPs by RNA and protein expression through the previous and the present studies. Our immunohistochemistry data revealed versican in the DPLTs, and ALP activity is significantly elevated in DPLTs than in ASCs. Moreover, the compact spheroid-forming structure of the cells was ob-

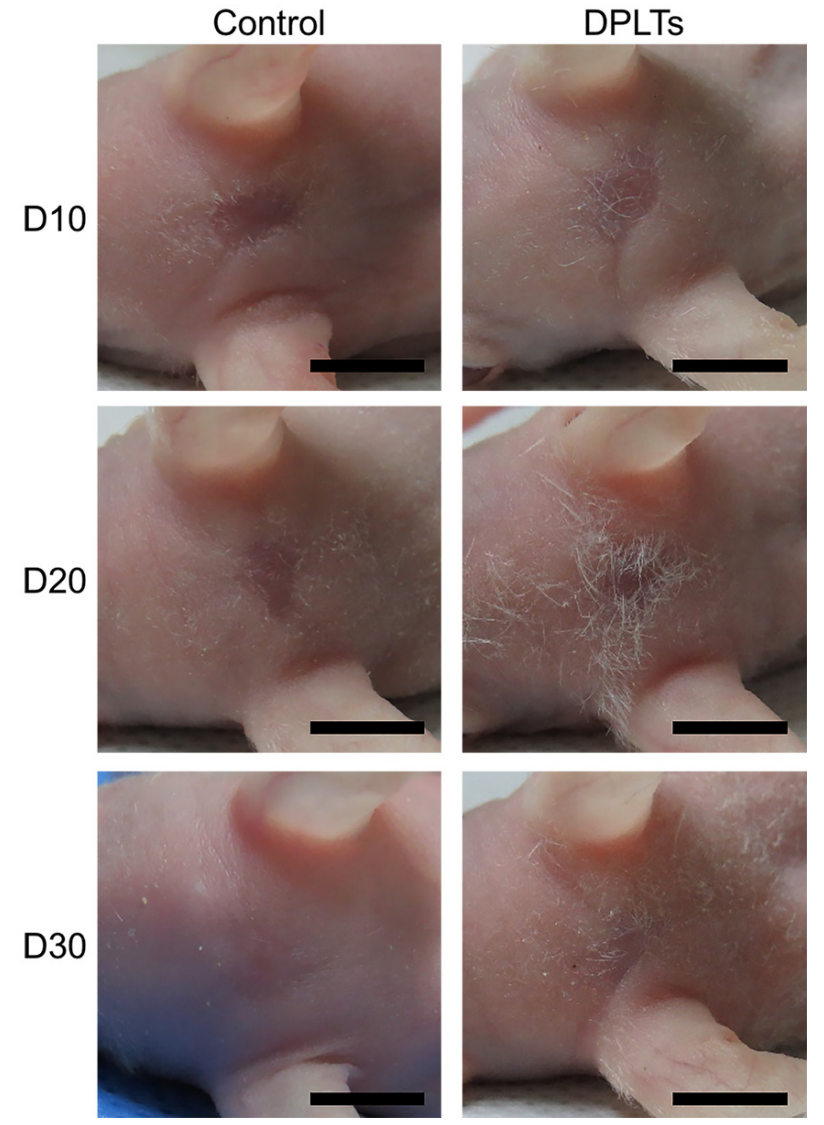

Fig. 2. Gross evaluation of hair growth in the wound bed. Photographs of the wounds at days 10,20 and 30 from the control and the DPLTs groups. Bar $=5 \mathrm{~mm}$. Hair growth around and at the wound bed was remarkable in the DPLTs group compared to the control group at day 20.

served in H\&E staining, and the extracellular matrix formed during aggregation of DPLTs was immunostained by laminin and vimentin. Versican and ALP are key characteristic molecular markers of DPs, which are specifically expressed in DPs during the anagen phase and absent in the telogen phase $[19,30]$. Vimentin and laminin represent the extracellular matrix present within the DPs [19]. These data suggest that DPLTs may have hair inducing activity. Consistent with our findings, the levels of versican and ALP are closely associated with in vivo hair inducing activity of the DPCs $[6,11,12]$, and a distinct tendency to form cell aggregates of dermal papilla cells indirectly indicates the in vivo trichogenic property of cells $[2,8,20]$.

In vivo trichogenesis was evaluated by injecting DPLTs into a full-thickness skin wound on the head of nude mice. Athymic nude mice, which were used in this study, are characterized by disturbed development of hair follicles and dysgenesis of the thymus [17, 18], which is a suitable animal model to evaluate hair regeneration with a xenograft of canine cells. Yoo et al. [32] transplanted DPLTs to innerdermis in nude mice with outer root sheath cells (ORSCs) as 

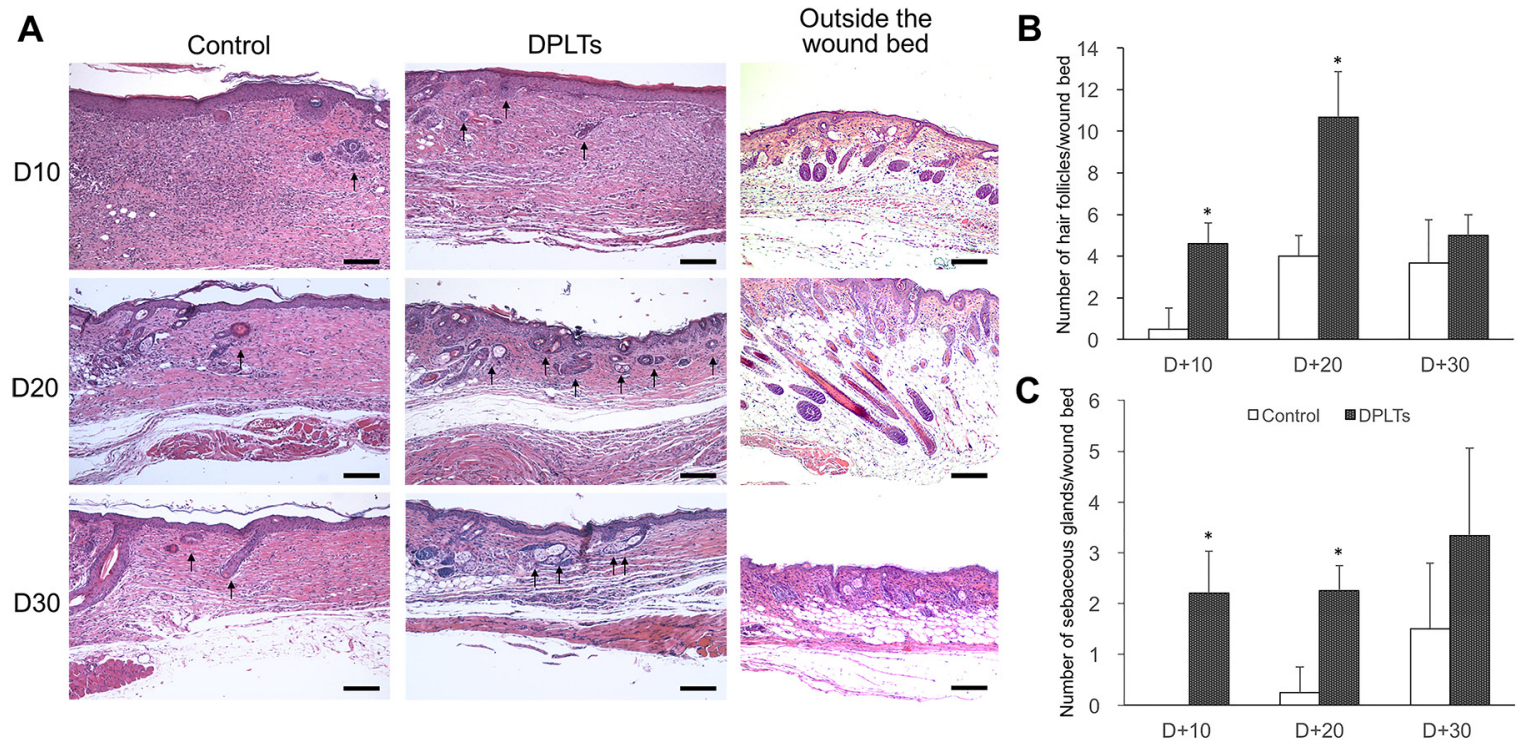

Fig. 3. Histological evaluation of the wound bed. Microphotographs of wounds and outside the wound bed at days 10, 20 and 30 (A). Regenerated hair follicles and sebaceous glands are indicated by arrows. H\&E stain; bar $=200 \mu \mathrm{m}$. Trichogenicity was assessed by the number of regenerated hair follicles (B) and regenerated sebaceous glands (C) per wound bed at a section. The number of regenerated hair follicle and sebaceous gland was significantly $\left({ }^{*} P<0.05\right)$ higher in the DPLTs group versus the control group at days 10 and 20 . At days 30 , the number of regenerated hair follicles was slightly decreased. And, between the groups, the differences in the number of hair follicles and sebaceous glands were unremarkable.
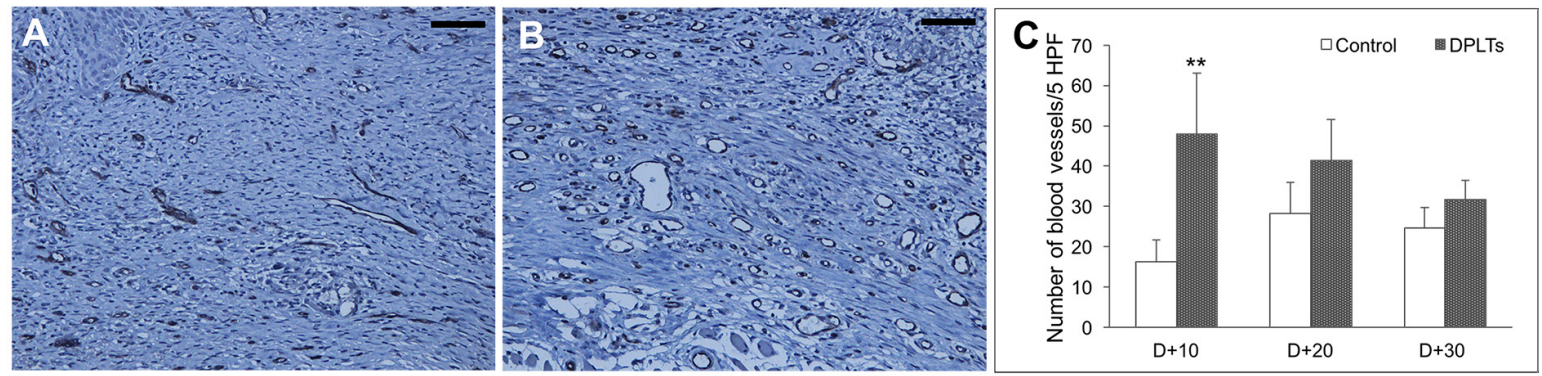

Fig. 4. Angiogenesis at the wound bed. Microphotographs of the wounds at 10 day in the control group (A) and the DPLTs group (B). IHC for CD31; bar $=100 \mu \mathrm{m}$. The number of vessels at the wound per five fields at days 10, 20 and 30 (C). Significantly $(* * P<0.01)$ more vessels were observed in wound bed from the DPLTs group than from the control group.

a source of epithelial cells. By contrast, we injected DPLTs as a mesenchymal component at the full-thickness skin wound, anticipating the wound epithelium would interact with dermal components as epithelial components as previously described [7]. Gross and microscopic findings at days 10 and 20 indicate that DPLTs play a crucial role in contributing to hair regeneration. Regeneration of sebaceous gland was evaluated with that of hair follicle in this study. The results of the present study indicated that not only hair follicles, but also the complete pilosebaceous units were regenerated by DPLTs. The number of hair follicles was slightly decreased at day 30 compared to that of at day 20, and the difference between the groups also decreased. There are two possible explanations for the decrease in regenerated hair follicles at day 30. First, hair inducing role of DPLTs could be influ- enced by the hair cycle of mice. Nude mice are not actually nude; they also have hair follicles and a hair cycle, which is synchronized and repeated with a 3-week interval [24]. This is consistent with our observation, and anagen phase proceeded during days 10-20, whereas at days 30, most hair follicles outside the wound bed were in the telogen phase, which was characterized by shrunk hair bulb. In addition, our findings demonstrated small amounts of injected DPLTs could survive for 30 days, and the effect of DPLTs was minimal at day 30 .

Moreover, in the histological examination, the number of vessels in the wound bed was higher in the DPLTs group. Especially, statistically significant difference between groups was observed at day 10, which suggests that implanting DPLTs on the injection site may enhance the neovasculariza- 
B

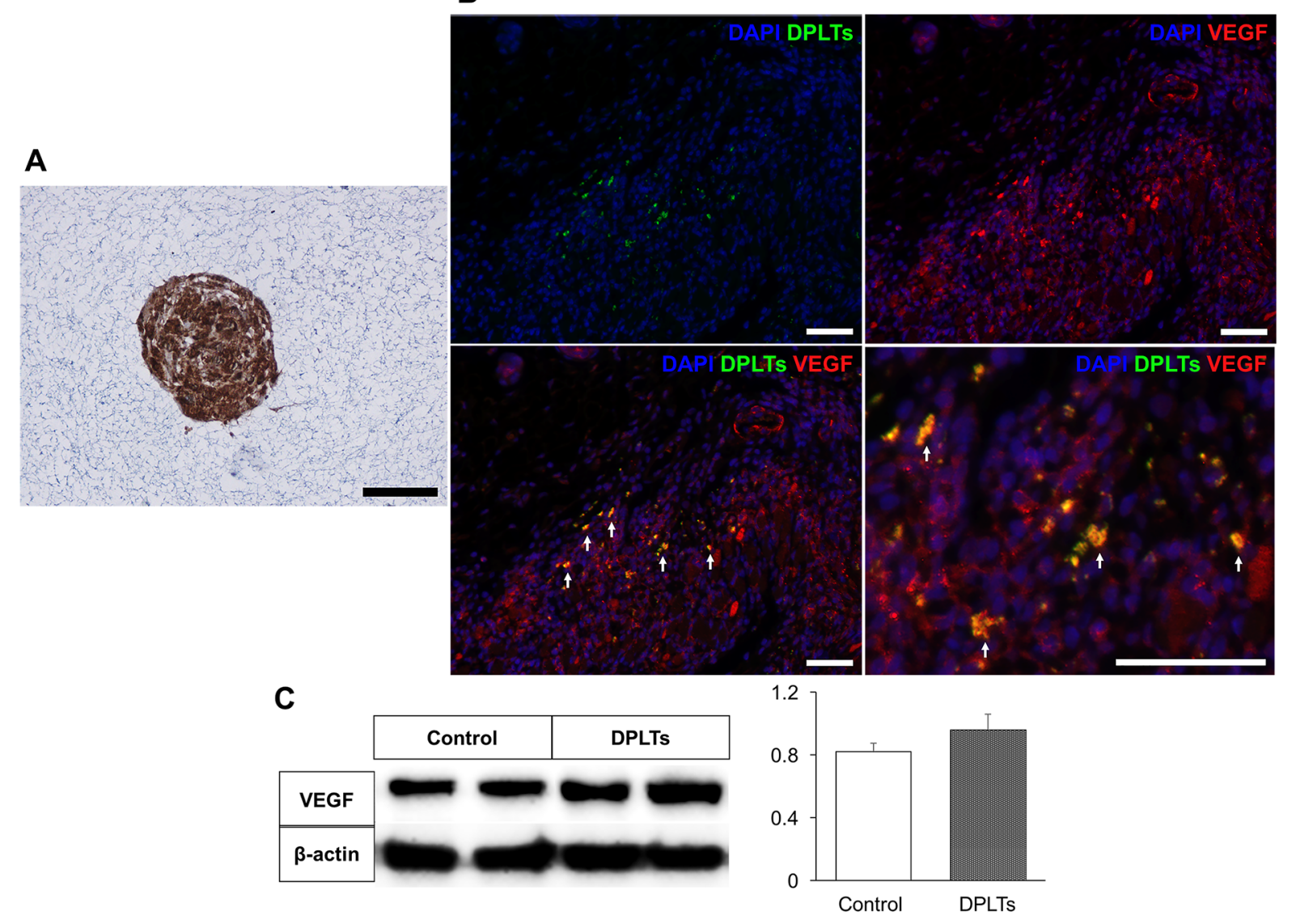

Fig. 5. In vitro (A) and in vivo (B) VEGF secretion of DPLTs and quantitative evaluation of VEGF from the wound at day 10 (C). (A) Notice strong positive cytoplasmic immunostaining for VEGF of collagen embedded DPLTs. IHC for VEGF; bar=50 $\mu \mathrm{m}$. (B) IHC of injected DPLTs (green-labeled) for VEGF (red) at 10 day. VEGF-positive DPLTs (yellow) are indicated by arrows. IHC for VEGF; bar $=100 \mu \mathrm{m}$. (C) Quantitative evaluation of VEGF protein from the wound at day 10 by Western blotting. Difference between groups was not significant.

tion. The decrease in the number of vessels in the DPLTs group could be explained by the decrease of granulation tissue as time passed. In addition, as the survival rate of injected DPLTs declined over time, the amount of VEGF secreted from DPLTs also diminished. The transition of the hair cycle (physiological hair loss) and alopecia (pathological hair loss) were not only controlled but also characterized by DPs and peri-papillary vascularization [23, 31]. In addition, the close connection between hair regeneration and neovascularization around hair follicles has been identified under various treatments for alopecia including when medical treatments and laser therapy were applied [13, 23, 29].

While mesenchymal stem cells have been observed to have a stimulatory effect on hair regeneration due to the paracrine secretion of growth factors in previous studies [10, 22], we expected DPLTs to be the direct structural components of the hair follicle as a substitute for DPs under control of EMI. Injected fluorescence-labeled DPLTs, however, did not constitute regenerated DPs. Therefore, EMI with reconstructed canine DPLTs is not fully evaluated in the present study, and a further study is needed. Instead of a direct structural role, growth factor secretion of DPLTs was evaluated in connection to the difference of vascularity among groups. Both embedded in collagen and injected DPLTs expressed VEGF. Although polyclonal antibody used in this study detected both murine and canine VEGF in an in vivo study, merged signals of DPLTs and VEGF could prove the presence of growth factors secreted from DPLTs. These results are consistent with the fact that the mesenchymal component, including DPCs, strongly expresses VEGF compared to other components of the hair follicle [14]. In other words, the VEGF autocrine effect of DPs is also identified on our reconstructed DPLTs. VEGF stimulates the proliferation of DPCs in vitro as well as stimulates hair regrowth and increases the size of the hair follicles and hair shafts in vivo $[15,31]$. As a result of Western blotting, the amount of VEGF tended to be higher in the DPLTs group than in the control group, although no significant difference was observed between groups. These results could be explained by the survival rate of DPLTs on wound bed. A further study and application of scaffold or 
biomaterials with DPLTs which act as a delivery vehicles and physical support, are needed in order to increase the bioavailability of them.

There are several limitations in the present study. First of all, structural role of DPLTs was not proved in the study, where activated epithelial cells through the wound healing had been expected to interact with DPLTs by EMI. A further study, where epithelial component should be injected with DPLTs (dermal component) without wound, was needed to evaluate EMI. Second, residual DPLTs and the amount of VEGF secreted by DPLTs were not constant by time according to our observation. The survival rate of cells on the injected site is the major concerns in regenerative medicine. Biomaterials which act as a scaffold for extending the survival time and maintaining 3D structure of DPLTs should be evaluated. Although only paracrine effect of DPLTs was proved in the present study, DPLTs also have the several positive effect on hair regeneration similar to DPs. The DPs have a stem cell-like role to differentiate into multi-lineage in itself, as well as the DPs contact the bulge and activate the bulge stem cells at the early anagen to form the the new hair follicles after remaining intact during the catagen and the telogen phases [5]. Furthermore, DPs help neighboring epidermal cells to differentiate into cells of hair follicle lineages $[5,30]$. Other paracrine and signaling pathways, such as Wnt/ $\beta$-catenin/BMP, FGF and IGF, in DPLTs also should be evaluated in a further study $[4,16,21]$.

In conclusion, this study demonstrates the possibility of DPLTs made from canine ASCs as a substitute for DPs in alopecic animals. In addition to the previous in vitro study, the in vivo results indicate that this engineered construct could enhance the hair follicle regeneration in an athymic nude mouse model by increasing neovascularization on the wound bed. To our knowledge, the development of reconstructed DPs from mesenchymal stem cells from animals has not been previously reported. Despite its imperfections, engineered canine DPLTs have the feasibility to be a useful treatment option for alopecic animals as an easily obtained source of DPCs as well as a study model to further characterize DPCs.

ACKNOWLEDGMENTS. This research was supported by the Basic Science Research Program of the National Research Foundation of Korea (NRF) and funded by the Ministry of Education, Science and Technology (2013-011357).

\section{REFERENCES}

1. Bae, S., Kim, J., Li, L., Lee, A., Lim, H., Jeong, J., Lee, S. H., Kweon, O. and Kim, W. H. 2015. Canine adipose-derived stem cell aggregates as a viable substitute to actual canine dermal papillae. Maced. Vet. Rev. 38: 1-6.

2. Bratka-Robia, C. B., Mitteregger, G., Aichinger, A., Egerbacher, M., Helmreich, M. and Bamberg, E. 2002. Primary cell culture and morphological characterization of canine dermal papilla cells and dermal fibroblasts. Vet. Dermatol. 13: 1-6. [Medline] [CrossRef]

3. Chueh, S. C., Lin, S. J., Chen, C. C., Lei, M., Wang, L. M., Widelitz, R., Hughes, M. W., Jiang, T. X. and Chuong, C. M.
2013. Therapeutic strategy for hair regeneration: hair cycle activation, niche environment modulation, wound-induced follicle neogenesis, and stem cell engineering. Expert Opin. Biol. Ther. 13: 377-391. [Medline] [CrossRef]

4. Danilenko, D. M., Ring, B. D. and Pierce, G. F. 1996. Growth factors and cytokines in hair follicle development and cycling: recent insights from animal models and the potentials for clinical therapy. Mol. Med. Today 2: 460-467. [Medline] [CrossRef]

5. Driskell, R. R., Clavel, C., Rendl, M. and Watt, F. M. 2011. Hair follicle dermal papilla cells at a glance. J. Cell Sci. 124: 1179-1182. [Medline] [CrossRef]

6. Iida, M., Ihara, S. and Matsuzaki, T. 2007. Hair cycle-dependent changes of alkaline phosphatase activity in the mesenchyme and epithelium in mouse vibrissal follicles. Dev. Growth Differ. 49: 185-195. [Medline] [CrossRef]

7. Jahoda, C. A. 1992. Induction of follicle formation and hair growth by vibrissa dermal papillae implanted into rat ear wounds: vibrissa-type fibres are specified. Development 115 : 1103-1109. [Medline]

8. Jahoda, C. A. and Oliver, R. F. 1984. Vibrissa dermal papilla cell aggregative behaviour in vivo and in vitro. J. Embryol. Exp. Morphol. 79: 211-224. [Medline]

9. Jahoda, C. A., Whitehouse, J., Reynolds, A. J. and Hole, N. 2003. Hair follicle dermal cells differentiate into adipogenic and osteogenic lineages. Exp. Dermatol. 12: 849-859. [Medline] [CrossRef]

10. Kim, H., Choi, K., Kweon, O. K. and Kim, W. H. 2012. Enhanced wound healing effect of canine adipose-derived mesenchymal stem cells with low-level laser therapy in athymic mice. J. Dermatol. Sci. 68: 149-156. [Medline] [CrossRef]

11. Kishimoto, J., Ehama, R., Wu, L., Jiang, S., Jiang, N. and Burgeson, R. E. 1999. Selective activation of the versican promoter by epithelial- mesenchymal interactions during hair follicle development. Proc. Natl. Acad. Sci. U.S.A. 96: 7336-7341. [Medline] [CrossRef]

12. Kobayashi, T., Fujisawa, A., Amagai, M., Iwasaki, T. and Ohyama, M. 2011. Molecular biological and immunohistological characterization of canine dermal papilla cells and the evaluation of culture conditions. Vet. Dermatol. 22: 414-422. [Medline] [CrossRef]

13. Kovacs, I. B., Mester, E. and Görög, P. 1974. Laser-induced stimulation of the vascularization of the healing wound. An ear chamber experiment. Experientia 30: 341-343. [Medline] [CrossRef]

14. Kozlowska, U., Blume-Peytavi, U., Kodelja, V., Sommer, C., Goerdt, S., Majewski, S., Jablonska, S. and Orfanos, C. E. 1998. Expression of vascular endothelial growth factor (VEGF) in various compartments of the human hair follicle. Arch. Dermatol. Res. 290: 661-668. [Medline] [CrossRef]

15. Lachgar, S., Moukadiri, H., Jonca, F., Charveron, M., Bouhaddioui, N., Gall, Y., Bonafe, J. L. and Plouët, J. 1996. Vascular endothelial growth factor is an autocrine growth factor for hair dermal papilla cells. J. Invest. Dermatol. 106: 17-23. [Medline] [CrossRef]

16. Lin, W. H., Xiang, L. J., Shi, H. X., Zhang, J., Jiang, L. P., Cai, P. T., Lin, Z. L., Lin, B. B., Huang, Y., Zhang, H. L., Fu, X. B., Guo, D. J., Li, X. K., Wang, X. J. and Xiao, J. 2015. Fibroblast growth factors stimulate hair growth through $\beta$-catenin and Shh expression in C57BL/6 mice. BioMed Res. Int. 2015: 730139. [Medline] [CrossRef]

17. Mecklenburg, L., Nakamura, M., Sundberg, J. P. and Paus, R. 2001. The nude mouse skin phenotype: the role of Foxn1 in hair follicle development and cycling. Exp. Mol. Pathol. 71: 171-178. [Medline] [CrossRef] 
18. Militzer, K. 2001. Hair growth pattern in nude mice. Cells Tissues Organs (Print) 168: 285-294. [Medline] [CrossRef]

19. Müntener, T., Doherr, M. G., Guscetti, F., Suter, M. M. and Welle, M. M. 2011. The canine hair cycle - a guide for the assessment of morphological and immunohistochemical criteria. Vet. Dermatol. 22: 383-395. [Medline] [CrossRef]

20. Osada, A., Iwabuchi, T., Kishimoto, J., Hamazaki, T. S. and Okochi, H. 2007. Long-term culture of mouse vibrissal dermal papilla cells and de novo hair follicle induction. Tissue Eng. 13: 975-982. [Medline] [CrossRef]

21. Ozeki, M. and Tabata, Y. 2003. In vivo promoted growth of mice hair follicles by the controlled release of growth factors. Biomaterials 24: 2387-2394. [Medline] [CrossRef]

22. Park, B. S., Kim, W. S., Choi, J. S., Kim, H. K., Won, J. H., Ohkubo, F. and Fukuoka, H. 2010. Hair growth stimulated by conditioned medium of adipose-derived stem cells is enhanced by hypoxia: evidence of increased growth factor secretion. Biomed. Res. 31: 27-34. [Medline] [CrossRef]

23. Popchristov, P., Konstantinov, A. and Obreshkova, E. 1968. The blood vessels of the scalp in patients with alopecia areata before and after corticosteroid therapy. Br. J. Dermatol. 80: 753-757. [Medline] [CrossRef]

24. Porter, R. M. 2003. Mouse models for human hair loss disorders. J. Anat. 202: 125-131. [Medline] [CrossRef]

25. Reynolds, A. J. and Jahoda, C. A. 1996. Hair matrix germinative epidermal cells confer follicle-inducing capabilities on dermal sheath and high passage papilla cells. Development 122:
3085-3094. [Medline]

26. Ryu, H. H., Lim, J. H., Byeon, Y. E., Park, J. R., Seo, M. S., Lee, Y. W., Kim, W. H., Kang, K. S. and Kweon, O. K. 2009. Functional recovery and neural differentiation after transplantation of allogenic adipose-derived stem cells in a canine model of acute spinal cord injury. J. Vet. Sci. 10: 273-284. [Medline] [CrossRef]

27. Schmidt-Ullrich, R. and Paus, R. 2005. Molecular principles of hair follicle induction and morphogenesis. BioEssays 27: 247-261. [Medline] [CrossRef]

28. Schneider, M. R., Schmidt-Ullrich, R. and Paus, R. 2009. The hair follicle as a dynamic miniorgan. Curr. Biol. 19: R132-R142. [Medline] [CrossRef]

29. Weiss, V. C., West, D. P., Fu, T. S., Robinson, L. A., Cook, B., Cohen, R. L. and Chambers, D. A. 1984. Alopecia areata treated with topical minoxidil. Arch. Dermatol. 120: 457-463. [Medline] [CrossRef]

30. Yang, C. C. and Cotsarelis, G. 2010. Review of hair follicle dermal cells. J. Dermatol. Sci. 57: 2-11. [Medline] [CrossRef]

31. Yano, K., Brown, L. F. and Detmar, M. 2001. Control of hair growth and follicle size by VEGF-mediated angiogenesis. $J$. Clin. Invest. 107: 409-417. [Medline] [CrossRef]

32. Yoo, B. Y., Shin, Y. H., Yoon, H. H., Seo, Y. K., Song, K. Y. and Park, J. K. 2010. Application of mesenchymal stem cells derived from bone marrow and umbilical cord in human hair multiplication. J. Dermatol. Sci. 60: 74-83. [Medline] [CrossRef] 